\title{
Acceleration rate of mitral inflow $E$ wave: a novel transmitral doppler index for assessing diastolic function
}

\author{
Roya Sattarzadeh ${ }^{1}$, Anahita Tavoosi ${ }^{*}$, Mostafa Jabbari ${ }^{1}$, Amir Farhang Zand Parsa', Babak Geraiely ${ }^{1}$,
} Mohammad Saadat ${ }^{1}$, Farnoosh Larti ${ }^{1}$, Ali Pasha Meysamie ${ }^{2}$ and Mehrdad Salehi ${ }^{1}$

\begin{abstract}
Background: We performed comprehensive transmitral and pulmonary venous Doppler echocardiographic studies to devise a novel index of diastolic function. This is the first study to assess the utility of the acceleration rate (AR) of the $\mathrm{E}$ wave of mitral inflow as a primary diagnostic modality for assessing diastolic function.

Methods: Study group consisted of 84 patients ( $53+11$ years) with left ventricle (LV) diastolic dysfunction and 34 healthy people ( $35 \pm 9$ years) as control group, who were referred for clinically indicated two-dimensional transthoracic echocardiogram (TTE) during 2012 and 2013 to Imam Hospital. Normal controls were defined as patients without clinical evidence of cardiac disease and had normal TTE. LV diastolic function was determined according to standardized protocol of American Society of Echocardiography (ASE). As our new parameter, AR of E wave of mitral inflow was also measured in all patients. It was represented by the slope of the line between onset of E wave and peak of it. Correlation between AR of E wave and LV diastolic function grade was measured using the Spearman correlation coefficient. Receiver operating characteristic (ROC) curve was used to determine the sensitivity and specificity of AR of E wave in diagnosing LV diastolic dysfunction in randomly selected two-thirds of population then its derived cutoff was evaluated in rest of the population. The institutional review board of the hospital approved the study protocol. All participants gave written informed consent. This investigation was in accordance with the Declaration of Helsinki.
\end{abstract}

Results: The mean value of AR was $1010 \pm 420 \mathrm{~cm} / \mathrm{s}^{2}$ in patients whereas the mean value for the normal controls was $701 \pm 210 \mathrm{~cm} / \mathrm{s}^{2}$. There was a strong and graded relation between AR of $E$ wave of mitral inflow and LV diastolic function grade (Spearman $P \leq 0.0001, r_{s}=0.69$ ). ROC curve analysis revealed that AR of $\mathrm{E}$ wave of mitral inflow $=750 \mathrm{~cm} / \mathrm{s}^{2}$ predicted moderate or severe LV diastolic dysfunction with $89 \%$ sensitivity and $89 \%$ specificity (area under curve $[\mathrm{AUC}]=0.903, P<0.0001$ ). Application of this cutoff on test group showed $96 \%$ sensitivity and $77 \%$ specificity with $A \cup C=0.932$ and $P<0.0001$.

Conclusion: The AR of E wave of mitral inflow could be used for assessment of diastolic function, especially moderate or severe diastolic dysfunction. However, before its clinical application, external validation should be considered.

\footnotetext{
* Correspondence: anahitatavoosi@gmail.com

${ }^{1}$ Cardiology Department of Imam Khomeini Hospital, Tehran University of

Medical Sciences, Tehran, Iran

Full list of author information is available at the end of the article
} 


\section{Background}

Over the past two decades, the prevalence of heart failure due to diastolic dysfunction has been gradually rising. Despite the growing incidence of this disorder, no effective therapies exist to treat the disease, halt its progression or reduce the associated mortality [1]. The assessment of left ventricular (LV) diastolic function and filling pressures is of paramount clinical importance to distinguish this syndrome from other diseases such as pulmonary disease resulting in dyspnea, to assess prognosis, and to identify underlying cardiac disease and its best treatment. LV filling pressures as measured invasively include mean pulmonary wedge pressure or mean left atrial (LA) pressure, and LV end-diastolic pressure [2, 3]. Echocardiography has played a central role in the evaluation of LV diastolic function over the past two decades. Transmitral Doppler echocardiography has been routinely used to identify left ventricular diastolic dysfunction in patients $[4,5]$. However, problems related to the complexity of interpreting the transmitral flow profile still exist, and some of the better established clinical indices may need to be re-evaluated for their relevance.

The estimation of LV filling pressures in patients with normal ejection fractions (EF)s is more challenging than in patients with depressed EFs. In this patient group, the ratio of mitral peak velocity of early filling (E) to early diastolic mitral annular velocity (e'), the E/e' ratio, should be calculated. An average ratio $\leq 8$ identifies patients with normal LV filling pressures, whereas a ratio $\geq 13$ indicates an increase in LV filling pressures. When the ratio is between 9 and 13, other measurements are essential. A pulmonary venous (PV) atrial reversal wave (Ar) duration longer than $30 \mathrm{~ms}$ of that of the transmitral A wave, a change in E/A ratio with the Valsalva maneuver of $\geq 0.5$, ratio between isovolumetric relaxation time (IVRT) and the time delay $\left(\mathrm{T}_{\mathrm{E}-\mathrm{e}}\right.$ ) between onset of mitral $\mathrm{E}$ and annular e,less than $2\left(\mathrm{IVRT} / \mathrm{T}_{\mathrm{E}-\mathrm{e}}<2\right)$, pulmonary artery systolic pressure $\geq 35 \mathrm{~mm} \mathrm{Hg}$ (in the absence of pulmonary disease), and maximal LA volume $\geq 34 \mathrm{~mL} / \mathrm{m}^{2}$ are all indicative of increased LV filling pressures. The presence of $\geq 2$ abnormal measurements increases the confidence in the conclusions [6]. By the way according to literature each of the mentioned criteria has some limitations which cause some difficulties in explanation of echocardiography results.

Therefore, we performed comprehensive transmitral and pulmonary venous Doppler echocardiographic studies to devise a novel index of diastolic function. This is the first study to assess the utility of the acceleration rate and time of the $\mathrm{E}$ wave of mitral inflow as a primary diagnostic modality for assessing diastolic function.

\section{Methods}

Study group consisted of 84 patients with LV diastolic dysfunction and 34 healthy people as control group, who were referred for clinically indicated two-dimensional transthoracic echocardiogram (TTE) between 2012 January and 2013 May. The inclusion criterion was presence of LV diastolic dysfunction in TTE. Patients with unstable hemodynamic state, arrhythmia, valvular heart disease, congenital heart disease, constrictive pericarditis or permanent pacemaker implantation were excluded. Normal controls were defined as patients without clinical evidence of cardiac disease and had normal TTE. Clinical data were obtained through a comprehensive review of patient's medical records.

The institutional review board of Imam Khomeini Hospital which is a tertiary hospital approved the study protocol. All participants gave written informed consent. This investigation was in accordance with the Declaration of Helsinki.

\section{Standard transthoracic echocardiography}

Complete M-mode, two-dimensional and Doppler echocardiogram was performed by two experienced cardiologists according to standardized protocol of American Society of Echocardiography $[7,8]$ using a commercially available instrument (VIVID 7, GE-Ving Med, Horten, Norway) equipped with a $3.5 \mathrm{MHz}$ transducer. Making use of the modified Simpson method, LV ejection fraction was measured at the apical four-chamber view.

\section{Assessment of diastolic function}

Mitral inflow was assessed from the apical 4-chamber view with pulsed wave Doppler by placing a $1-2 \mathrm{~mm}$ sample volume between the tips of the mitral leaflets during diastole. From the mitral inflow profile, the Eand A-wave velocity, E-deceleration time (DT), A-wave duration, and $E / A$ velocity ratio were measured. Pulmonary venous velocities were obtained from the same window with the sample volume placed $1 \mathrm{~cm}$ into the right upper pulmonary vein. The flow velocities were recorded, the ratio of systolic to diastolic flow ( $S / D$ ratio) was calculated and duration of atrial reversal flow was measured. Doppler tissue imaging was used to measure $E^{\prime}$ and $A^{\prime}$ velocities by placing a $1-2 \mathrm{~mm}$ sample volume in the septal and lateral mitral annulus.

LV diastolic function was determined using standard echocardiographic parameters including $E / A$ velocity ratio, E-DT, PV atrial reversal velocity and duration, PV $S / D$ ratio, and mitral $E / E^{\prime}$ ratio. Diastolic function is graded as normal, abnormal relaxation (Grade I), pseudonormal (Grade II), and restrictive (Grade III) as described previously. Pseudonormal (Grade II) is differentiated from normal by having (i) PV atrial reversal duration longer than mitral A duration by $30 \mathrm{~ms}$; or (ii) peak PV atrial reversal velocity $>35 \mathrm{~cm} / \mathrm{s}$; or (iii) mitral $E / E^{\prime}>12$ (lateral annulus) or $>15$ (septal annulus) $[2,6]$. 
Acceleration rate of $E$ wave of mitral inflow (AR)

As our new parameter acceleration rate and time of $E$ was also measured in all patients. Acceleration rate of $\mathrm{E}$ $(\mathrm{cm} / \mathrm{sec} 2)$ was represented by the slope of the line between an anchored point and a crosshair (Fig. 1). This linear measurement was made on the velocity spectrum. Acceleration time (AT) of E was measured from onset to peak of $E$ These recordings were shown on a strip chart with a sweep speed of $100 \mathrm{~mm} / \mathrm{s}$ to determine correct temporal observations. Measurements were performed off line by an independent observer who had no knowledge of the Doppler or Tissue Doppler findings. At least three measurements were taken of each parameter and these were averaged.

\section{Statistical analysis}

SPSS release 21.0 statistical package was used for data analysis. All values were expressed as mean \pm SD. Correlation between AR and AT of E wave and LV diastolic function grade was measured using the Spearman correlation coefficient. Multivariate Logistic Regression analysis was also done to adjust the age and gender effect. Receiver operating characteristic (ROC) curve was used to determine the sensitivity and specificity of AR of $E$ wave in diagnosing LV diastolic dysfunction and elevated left ventricle diastolic pressure (LVDP). Study population randomly assigned into two groups with $2: 1$ ratio. ROC curve was performed in randomly selected two-thirds of population (derivation group), then the derived cutoff was evaluated in the rest of the population (Test group). Therefore, ROC curve analysis was performed two times. First, we recoded the "diastolic function" into a dichotic variable just based on presence or absence of any degree of diastolic dysfunction (mild, moderate, and severe). Second, we recoded the "diastolic function" into another dichotic variable, this time based on presence or absence of elevated LVDP (moderate and severe diastolic dysfunction).

\section{Result}

The study population consisted of 84 adult patients (64\% men), mean age $53 \pm 11$ (range, $20-70$ years) and 34 normal controls, who were referred for clinically indicated two-dimensional echocardiogram. They were referred because of the following reasons: dyspnea/peripheral edema/ congestive heart failure (48\%), cerebrovascular accident (12\%), preoperative assessment (10\%), coronary artery disease ( $9 \%)$, and others (21\%).

The baseline clinical and echocardiographic characteristics are listed in Table 1.

The mean value of $\mathrm{E}$ acceleration time and $\mathrm{E}$ acceleration rate were $85.6 \pm 19.1 \mathrm{~ms}$, and $1010 \pm 420 \mathrm{~cm} / \mathrm{s}^{2}$ in case group respectively, whereas these mean values for the normal controls were $96.7 \pm 15.1 \mathrm{~ms}$, and $701 \pm$ $210 \mathrm{~cm} / \mathrm{s}^{2}$ respectively. The mean values of AT and AR according to grade of LV diastolic dysfunction is showed in Table 2. There was a strong and graded relation between AR of $\mathrm{E}$ wave of mitral inflow and LV diastolic function grade (Spearman $P \leq 0.0001, \mathrm{r}_{\mathrm{s}}=0.69$ ) (Fig. 2). The Logistic Regression Analysis analysis showed that AR of $\mathrm{E}$ could predict the diastolic dysfunction after adjustment for age and gender $(P=0.001)$. Receiver operating characteristic (ROC) curve analysis in "derivation group" revealed that $A R$ of $E$ wave of mitral inflow $=655 \mathrm{~cm} / \mathrm{s}^{2}$ predicted presence of LV diastolic dysfunction with $71 \%$ sensitivity and $65 \%$ specificity (area under curve [AUC] $=$ 0. 0.727, $P=0.003)$. Application of this cutoff on test group showed $84 \%$ sensitivity and $64 \%$ specificity with AUC $=0.800$ and $P=0.001$. When considering only Grade II and III (moderate and severe) diastolic dysfunction, an AR of $750 \mathrm{~cm} / \mathrm{s}^{2}$ predicted at least moderate diastolic dysfunction with $89 \%$ sensitivity and $89 \%$ specificity (area

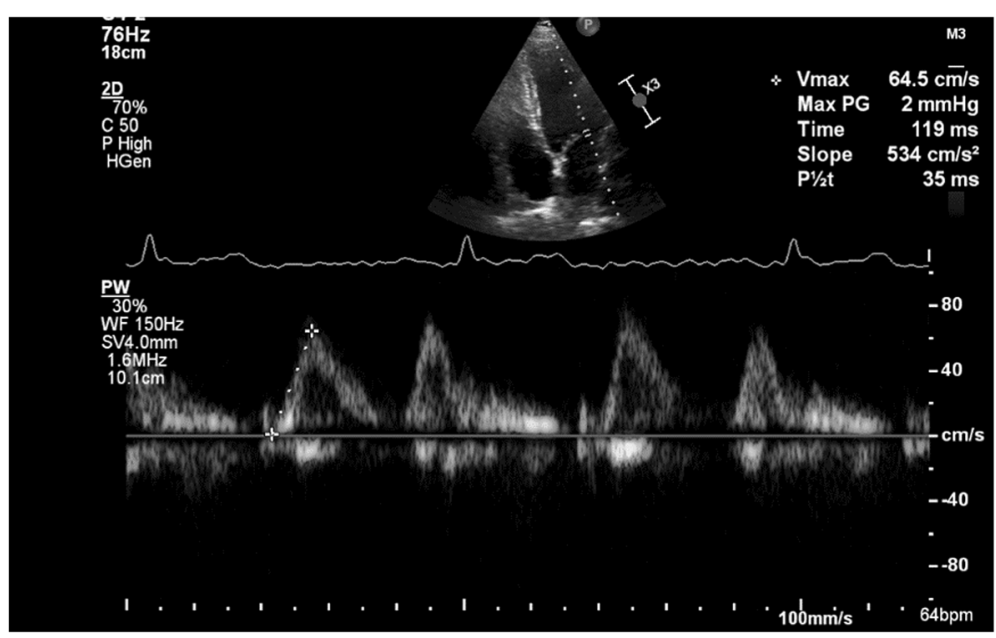

Fig. 1 Acceleration rate of $E$ wave of mitral inflow 
Table 1 Echocardiographic parameters of the study population

\begin{tabular}{llllll}
\hline & Normal $(n=34)$ & Grade I $(n=24)$ & Grade II $(n=39)$ & Grade III $(n=21)$ & Total $(n=118)$ \\
\hline Age (year) & $34.6 \pm 9.1$ & $53.2 \pm 7.2$ & $54.7 \pm 11.7$ & $48.9 \pm 12.9$ & $47.5 \pm 13.4$ \\
Men (\%) & 82 & 46 & 69 & 76 & 69 \\
Left ventricle ejection fraction (Simpson) (\%) & $55.7 \pm 4$ & $52.4 \pm 4.3$ & $37.9 \pm 2.9$ & $27.6 \pm 12.4$ & $44.3 \pm 13.8$ \\
Mitral peak E velocity (m/s) & $0.7 \pm 0.1$ & $0.50 \pm 0.1$ & $0.7 \pm 0.2$ & $1.04 \pm 0.2$ & $0.7 \pm 0.2$ \\
Mitral peak A velocity (m/s) & $0.5 \pm 0.09$ & $0.7 \pm 0.1$ & $0.6 \pm 0.1$ & $0.3 \pm 0.1$ & $0.6 \pm 0.2$ \\
Mitral E/A ratio & $1.4 \pm 0.3$ & $0.7 \pm 0.1$ & $1.2 \pm 0.4$ & $2.9 \pm 0.7$ & $1.5 \pm 0.8$ \\
Mitral deceleration time (ms) & $182 \pm 30$ & $236 \pm 23$ & $170 \pm 51$ & $108 \pm 38$ & $176 \pm 55$ \\
Pulmonary venous systolic forward flow velocity (m/s) & $0.66 \pm 0.08$ & $0.72 \pm 0.12$ & $0.54 \pm 0.17$ & $0.35 \pm 0.14$ & $0.58 \pm 0.18$ \\
Pulmonary venous diastolic forward flow velocity (m/s) & $0.54 \pm 0.08$ & $0.45 \pm 0.07$ & $0.64 \pm 0.18$ & $0.75 \pm 0.23$ & $0.59 \pm 0.18$ \\
Pulmonary venous atrial reversal velocity (m/s) & $0.23 \pm 0.03$ & $0.27 \pm 0.05$ & $0.36 \pm 0.08$ & $0.37 \pm 0.5$ & $0.30 \pm 0.08$ \\
Tissue Doppler imaging septal E' (cm/s) & $10.5 \pm 2.2$ & $6 \pm 0.9$ & $6 \pm 2.2$ & $5.1 \pm 1.7$ & $7.1 \pm 2.9$ \\
Tissue Doppler imaging lateral E' (cm/s) & $13.4 \pm 3.0$ & $8.2 \pm 1.7$ & $7.3 \pm 3.0$ & $6.8 \pm 2.2$ & $9.1 \pm 3.8$ \\
Tissue Doppler imaging septal A' (cm/s) & $7.6 \pm 1.6$ & $9.5 \pm 1.6$ & $6.7 \pm 2.4$ & $3.8 \pm 1.7$ & $7 \pm 2.6$ \\
Tissue Doppler imaging lateral A' (cm/s) & $7.3 \pm 2.2$ & $10.3 \pm 2.3$ & $5.7 \pm 2.3$ & $3.5 \pm 1.1$ & $6.7 \pm 3.1$ \\
E/E' & $7.2 \pm 1.7$ & $9.7 \pm 2.5$ & $13.9 \pm 5.6$ & $22.3 \pm 8.2$ & $12.6 \pm 7.2$ \\
Color M-mode flow propagation velocity (cm/s) & $61.9 \pm 7.9$ & $49.8 \pm 3.4$ & $41.6 \pm 6.6$ & $36.5 \pm 6.1$ & $48.4 \pm 11.5$ \\
Left atrium volume (cm ${ }^{3}$ ) & $42 \pm 11.7$ & $46.2 \pm 9.2$ & $88.4 \pm 28.3$ & $107 \pm 29.7$ & $69.8 \pm 24.2$ \\
\hline & & & &
\end{tabular}

under curve 0.903, $P<0.0001$ ) (Fig. 3, Table 3). Application of this cutoff on test group showed $96 \%$ sensitivity and $77 \%$ specificity with AUC $=0.932$ and $P<0.0001$. AR of $\mathrm{E}=1250 \mathrm{~cm} / \mathrm{s}^{2}$ was $100 \%$ specific for the detection of elevated LVDP (moderate or severe diastolic dysfunction) but with a low sensitivity of $22 \%$.

\section{Discussion}

The present study showed that AR of E wave of mitral inflow has a strong and graded relation to the LV diastolic function grade, and could be used especially to identify moderate or severe LV diastolic dysfunction. This result was predictable because according to Newton's second law there is a direct relationship between acceleration and pressure. E wave acceleration is directly determined by LA pressure and inversely related to myocardial relaxation [9]. Thomas and weyman [10] demonstrated in a mathematical model of LV filling that AR is greatly affected by changes in left atrial pressure compared with peak velocity, peak deceleration, and the total integral of the inflow velocity.

Despite the theoretical observations by Thomas and Weyman [10], AR has not been used clinically for estimation of LA pressure. There are two studies which reported AT and AR as parameters which can be useful for assessing left ventricular diastolic function in patients with diabetes or coronary artery diseases [11, 12]. There is also an experimental study on mice which reported AT of E wave was sensitive for detecting early stages of diastolic function, and appeared to add incremental value over that provided by the E/A ratio and IVRT for detecting later stages of diastolic dysfunction in murine models [13]. To our knowledge none of these studies reported a cut of value for AR of $\mathrm{E}$ wave to predict LV diastolic dysfunction. However in 1996 Nagueh et al. showed that peak AR $\geq 1900 \mathrm{~cm} / \mathrm{s}^{2}$ had a $77 \%$ sensitivity and $94 \%$ specificity for left ventricle diastolic pressure (LVDP) $>15 \mathrm{mmHg}$ in patients with atrial fibrillation(AF) [14]. In our study we showed that by simple measurement of AR of $E$ of mitral valve we might be able to identify patients with elevated LVDP. This application could be clinically important and valuable because it might explain patients' symptoms in some conditions. The cut off value of $750 \mathrm{~cm} / \mathrm{s}^{2}$ had a suitable sensitivity and specificity for elevated LVDP according to diastolic function grade II or more. And the cut off value of $1250 \mathrm{~cm} / \mathrm{s}^{2}$ had $100 \%$ specificity for diastolic dysfunction of grade II or more. The difference in our cut off value and the previous one could have three reasons. First, in our study we used AR which conventionally could be measured by

Table 2 Acceleration rate and time of $\mathrm{E}$ wave by diastolic function grade

\begin{tabular}{llllll}
\hline & Normal $(n=34)$ & Grade I $(n=24)$ & Grade II $(n=39)$ & Grade III $(n=21)$ & Total $(n=118)$ \\
\hline Acceleration time $(\mathrm{ms})$ & $96.7 \pm 15.1$ & $95.7 \pm 16.7$ & $83 \pm 18.9$ & $79.1 \pm 18.5$ & $88.8 \pm 18.7$ \\
Acceleration rate $\left(\mathrm{cm} / \mathrm{s}^{2}\right)$ & $701 \pm 210$ & $620 \pm 90$ & $1060 \pm 330$ & $1400 \pm 440$ & $930 \pm 400$ \\
\hline
\end{tabular}




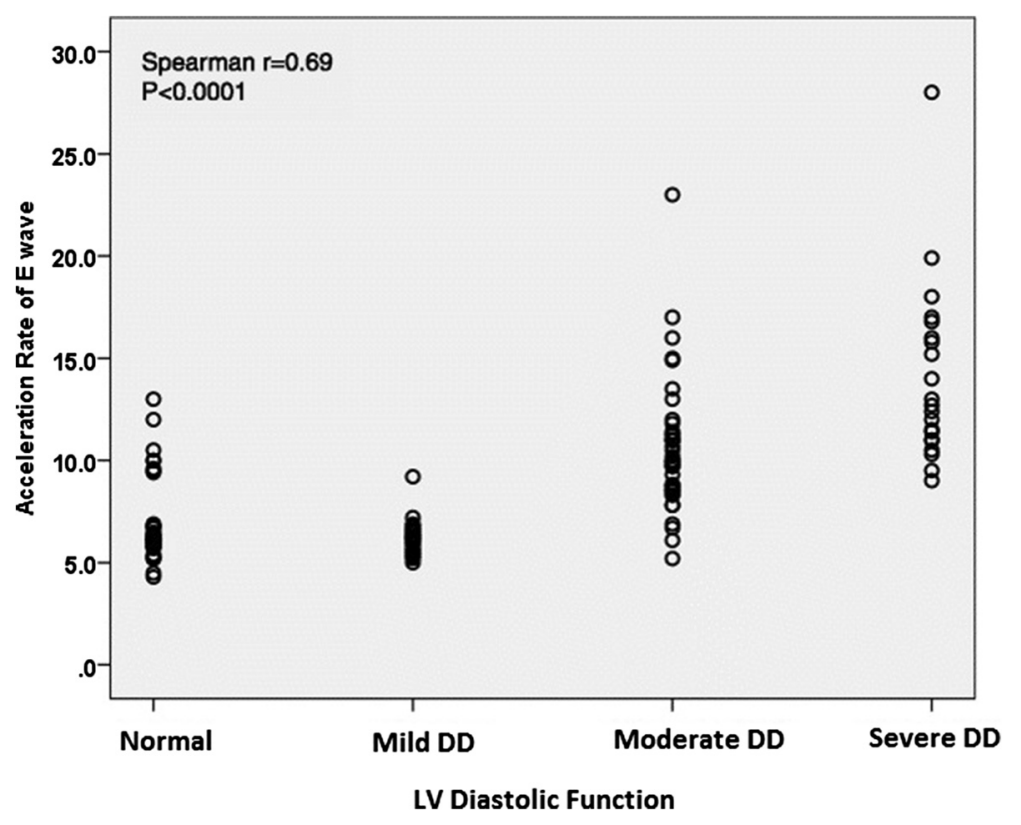

Fig. 2 Relationship between acceleration rate of E wave of mitral inflow and LV diastolic function grade

every machine of echocardiography, but Naughueh et.al used a computer software to measure peak of AR. Second the method of estimation of elevated LVDP was different in two studies. In our study we postulated that presence of diastolic dysfunction of grade II or more would be associated with elevated LVDP, however Naughueh et al. directly measured LVDP or LA pressure in their patients. Third,

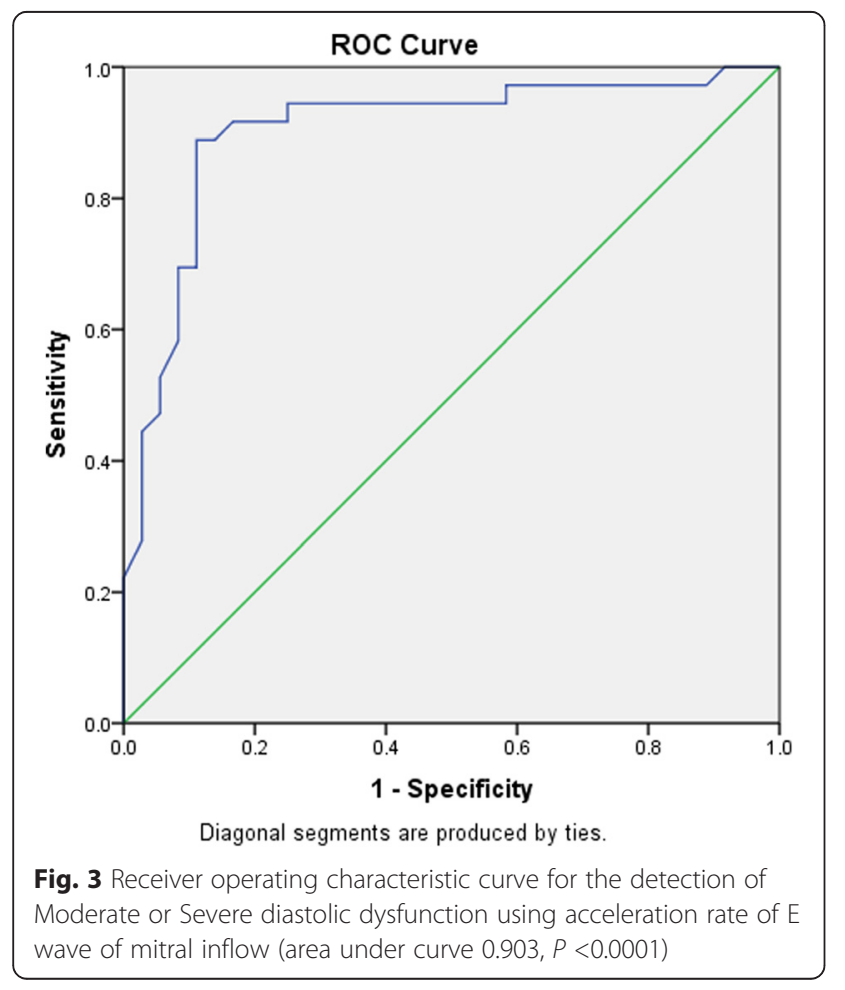

all of our patients had sinus rhythm, but the rhythm of patients in that study was AF.

\section{Limitation}

The limitations of our study include its relatively small size. The 'normal controls' are included on the basis of absence of a history of cardiovascular disease and a normal resting two-dimensional echocardiogram. Stress tests were not performed to rule out occult coronary artery disease. We used previously published Doppler echocardiographic referenced standards to define the different grades of LV diastolic function. Cardiac catheterization was not performed to evaluate LV diastolic function. However, these reference standards were previously validated with cardiac catheterization and are widely accepted as standards for classification of LV diastolic function grade. Although the results of our study about AR are promising, before its clinical application external validation should be considered. Further studies with invasive haemodynamic measurements are needed to show if AR measurement increase the

Table 3 Positive Predictive Value (PPV), Negative Predictive Value (NPV), sensitivity and specificity of acceleration rate of $E$ to detect moderate or severe diastolic dysfunction

\begin{tabular}{lllll}
\hline \multicolumn{3}{c}{$\begin{array}{l}\text { Moderate or severe diastolic } \\
\text { dysfunction }\end{array}$} & \\
\cline { 2 - 4 } & No & Yes & NPV $=89 \%$ \\
\hline Acceleration rate of E & $<750$ & 32 & 4 & PPV $=89 \%$ \\
& $\geq 750$ & 4 & 32 & \\
& & Spe $=89 \%$ & Sen $=89 \%$ & \\
\hline
\end{tabular}


diagnostic accuracy of LV diastolic dysfunction and elevated LA pressure with respect to validated echo-Doppler parameters.

\section{Conclusion}

AR of EARE wave of mitral inflow could be used for assessment of diastolic function, especially moderate to severe diastolic dysfunction. The cutoff value of $750 \mathrm{~cm} / \mathrm{s} 2$ could deserve as suitable cutoff point with $89 \%$ sensitivity and $89 \%$ specificity in detection of moderate to severe diastolic dysfunction. Before its clinical application external validation should be considered.

\section{Acknowledgements}

\section{Authors' contributions}

Roya Sattarzadeh was responsible for idea formation, study designing, performing echocardiography and reviewing the article. Anahita Tavoosi contributed in idea formation, study designing, performig echocardiography,drafting and reviewing the article. Mostafa Jabbari was responsible for data gathering and helped in analysis and drafting the article. Amir Farhang Zand Parsa, Babak Geraiely and Mehrdad Salehi were responsible for critical review and editing the manuscript. Mohammad Saadat and Farnoosh Larti had significant contribution in revision of the article by performing requested analysis and rewriting some parts of the manuscript. Alipasha Meysami contributed by consultation about study designing, and analysis of the data. All authors read and approved the final manuscript

\section{Competing interests}

We also confirm that this study complied with the principles of the declaration of Helsinki. Study protocol was approved by Local Ethics Committee of Tehran University of Medical Sciences. Informed consent was obtained from all physicians participated in this study. No conflict of interest existed for this study. No fund or grant was received to support this study.

\section{Author details}

${ }^{1}$ Cardiology Department of Imam Khomeini Hospital, Tehran University of Medical Sciences, Tehran, Iran. ${ }^{2}$ Department of community medicine, Tehran University of Medical Sciences, Tehran, Iran.

Received: 21 January 2016 Accepted: 4 June 2016

Published online: 10 June 2016

\section{References}

1. Nishimura RA, Jaber W. Understanding "diastolic heart failure": the tip of the iceberg. J Am Coll Cardiol. 2007;49(6):695-7.

2. Dokainish H, Nguyen JS, Bobek J, Goswami R, Lakkis NM. Assessment of the American Society of Echocardiography-European Association of Echocardiography guidelines for diastolic function in patients with depressed ejection fraction: an echocardiographic and invasive haemodynamic study. Eur J Echocardiogr. 2011;12(11):857-64.

3. Little WC, Oh JK. Echocardiographic evaluation of diastolic function can be used to guide clinical care. Circulation. 2009;120(9):802-9.

4. Appleton CP, Hatle LK, Popp RL. Relation of transmitral flow velocity patterns to left ventricular diastolic function: new insights from a combined hemodynamic and Doppler echocardiographic study. J Am Coll Cardiol. 1988;12(2):426-40.

5. Tabata T, Thomas JD, Klein AL. Pulmonary venous flow by doppler echocardiography: revisited 12 years later. J Am Coll Cardiol. 2003;41(8):1243-50.

6. Nagueh SF, Appleton CP, Gillebert TC, Marino PN, Oh JK, Smiseth OA, et al. Recommendations for the evaluation of left ventricular diastolic function by echocardiography. J Am Soc Echocardiogr. 2009;22(2):107-33.

7. Lang RM, Bierig M, Devereux RB, Flachskampf FA, Foster E, Pellikka PA, et al. Recommendations for chamber quantification: a report from the American Society of Echocardiography's Guidelines and Standards Committee and the Chamber Quantification Writing Group, developed in conjunction with the European Association of Echocardiography, a branch of the European Society of Cardiology. J Am Soc Echocardiogr. 2005;18(12):1440-63.
8. Lang RM, Badano LP, Mor-Avi V, Afilalo J, Armstrong A, Ernande L, et al. Recommendations for cardiac chamber quantification by echocardiography in adults: an update from the American Society of Echocardiography and the European Association of Cardiovascular Imaging. J Am Soc Echocardiogr. 2015;28(1):1-39. e14.

9. Ohno M, Cheng CP, Little WC. Mechanism of altered patterns of left ventricular filling during the development of congestive heart failure. Circulation. 1994; 89(5):2241-50.

10. Thomas JD, Weyman AE. Echocardiographic Doppler evaluation of left ventricular diastolic function. Physics and physiology. Circulation. 1991;84(3):977-90.

11. Bajraktari G, Qirko S, Fusco R, Milazzo A, Xhaxho B, Pezzano A. Transmitral pulsed-Doppler echocardiography is a more accurate technique compared with two-dimensional echocardiography using dobutamine, in patients with one vessel coronary artery disease. Eur J Heart Fail. 2003;5(1):63-72.

12. Riordan MM, Chung CS, Kovacs SJ. Diabetes and diastolic function: stiffness and relaxation from transmitral flow. Ultrasound Med Biol. 2005;31(12):1589-96.

13. Yuan L, Wang T, Liu F, Cohen ED, Patel W. An evaluation of transmitral and pulmonary venous Doppler indices for assessing murine left ventricular diastolic function. J Am Soc Echocardiogr. 2010;23(8):887-97. Pubmed Central PMCID: 2910830.

14. Nagueh SF, Kopelen HA, Quinones MA. Assessment of left ventricular filling pressures by Doppler in the presence of atrial fibrillation. Circulation. 1996; 94(9):2138-45
Submit your next manuscript to BioMed Central and we will help you at every step:

- We accept pre-submission inquiries

- Our selector tool helps you to find the most relevant journal

- We provide round the clock customer support

- Convenient online submission

- Thorough peer review

- Inclusion in PubMed and all major indexing services

- Maximum visibility for your research

Submit your manuscript at www.biomedcentral.com/submit
C Biomed Central 